\title{
STAR CLUSTERS IN QUASARS: Bloated Stars As Broad Emission Line Clouds
}

\author{
R.C. Puetter \\ The Center for Astrophysics and Space Sciences \\ The University of California, San Diego \\ La Jolla, CA 92093
}

\section{INTRODUCTION}

Many scenarios of the evolution of star clusters in the centers of galaxies involve the formation of a central supermassive object. Since black hole formation is not $100 \%$ efficient in mass usage, stars are left over. This paper investigates the properties of such stars and proposes that their externally heated atmospheres become "bloated" due to radiative forces from trapped line radiation. Such stars would swell to many times their normal diameters and acquire densities, sizes, and mean column masses typical of QSO/AGN emission line clouds (ELCs).

This idea is not new, and has been discussed earlier by several authors (e.g. Fabian 1979, Edwards 1980). However, the idea of "bloated" stars has recently received more attention (Puetter 1986, 1988, Penston 1988, Voit and Shull 1988), perhaps partly due to the demonstration (Peterson et al. 1985; Gondhalekar et al. 1986; Zheng et al. 1987) that many objects have ELCs with densities comparable to stellar chromospheres $\left(10^{12} \mathrm{~cm}^{-3}\right)$, and partly due to the realization that bloated stars could solve many problems, including (1) ELC confinement, (2) the narrow range of ELC column mass, density, and size, (3) the source ELC mass, (4) how ELCs avoid Keplerian shear disruption during orbital motion, and (5) how the ELCs maintain a small internal velocity dispersion while the ensemble motion approaches the speed of light.

\section{THE CALCULATION}

The basic motivation for proposing radiatively bloated stars is found in the recent calculations of Ferland and Elitzur (Ferland and Elitzur 1984; Elitzur and Ferland 1986). These authors demonstrate that diffuse radiation pressure can greatly exceed the gas pressure. For standard ELCs this is ruled out since the clouds would be disrupted. However, due to gravity, bloated stars would be stable under such circumstances.

In order to explore the plausibility of bloated stars, I have constructed a simple model. I assume the bloated atmosphere is (1) isothermal, (2) illuminated from all sides by powerlaw radiation, and (3) supported by line radiation with an optical depth dependence given by Ly $\alpha$-i.e. $P_{\text {rad }}$ is equal to $10 \times P_{\text {rad }}($ Ly $\alpha)$ to account for the radiative forces from all lines. In order to calculate the radiative forces, I have adopted a two level scaling law solution to the radiation transfer in Ly $\alpha$. A self-consistent, simultaneous solution of the momentum equation, the mass conservation equation, and ionization equation is then derived.

While Ly $\alpha$ dominates ELC radiation pressure (it saturates and is optically thick enough for appreciable spectral band width), it does not dominate the internal radiative forces. It is $\nabla P_{\text {rad }}$ that provides the radiative force. Hence once a line 

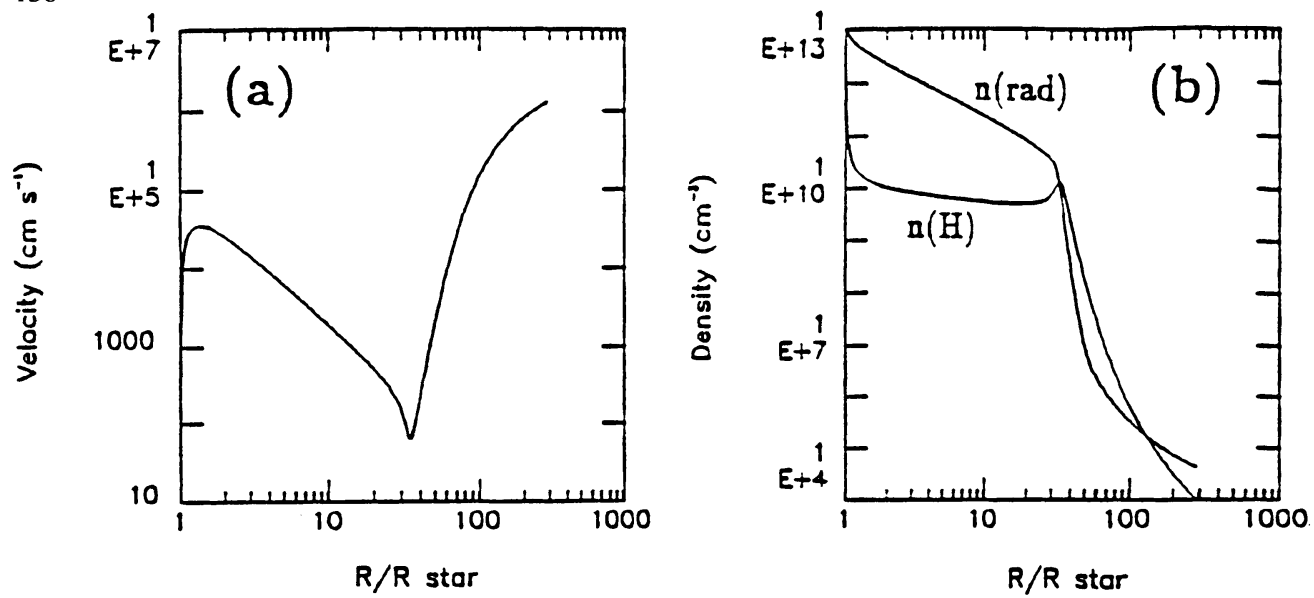

saturates, $\nabla P_{\text {rad }}$ is small since spectral band width grows slowly. Thus the Balmer lines, the $F e I I$ lines, etc., should contribute as much to $\nabla P_{\text {rad }}$ as does $L y \alpha$. Indeed, $\nabla P_{\text {rad }}$ calculated for the deep portion of the model presented here may significantly underestimate the true value since $L y \alpha$ becomes saturated.

Figure 1 presents the results. The adopted temperature is $2 \times 10^{4} \mathrm{~K}$, i.e. a temperature appropriate to the $L y \alpha$ forming region. This temperature also produces a source function appropriate to the other lines. The external radiation source is a $\nu^{-1}$ powerlaw with an intensity of $10^{-7} \mathrm{ergs}^{-1} \mathrm{~cm}^{-2} \mathrm{~Hz}^{-1}$ at 1 Rydberg $(\Gamma \approx 0.005)$. Panel (a) plots the flow velocity versus stellar radius. The stellar wind makes the subsonic to supersonic transition $\left(v_{s}=13 \mathrm{~km} \mathrm{~s}^{-1}\right)$ at the isothermal Parker radius $\left(r_{p}=\mathrm{GM} m_{p} / 2 \mathrm{kT}=4 \times 10^{13} \mathrm{~cm}\right)$. Below the Parker radius the solution is subsonic, going to zero velocity at the photosphere. Thus the bulk flow does not affect the line transfer (i.e. a Sobolev-type treatment is not required) and the internal velocity dispersion is given by the thermal Doppler width.

Panel (b) plots the total hydrogen density, $\mathrm{n}(\mathrm{H})$, and the equivalent "density due to radiation", $n_{\text {rad }}\left(\equiv P_{\text {rad }} / \mathrm{kT}\right)$, versus $\mathrm{R}$. As can be seen, the radiation pressure gradient lifts a high density $\left(n \approx 10^{10} \mathrm{~cm}^{-3}\right)$ shell to a distance of roughly $40 R_{\odot}$ (i.e. $3 \times 10^{12} \mathrm{~cm}$ ). The resulting column density, excluding the stellar core, is $6 \times 10^{22} \mathrm{~cm}^{-2}$.

\section{REFERENCES}

Edwards, A.C. 1980, M.N.R.A.S., 190, 757.

Elitzur, M., and Ferland, G. 1986, Ap. J., 305, 35.

Fabian, A.C. 1979, Proc. R. Soc. Lond., A, 366, 449.

Ferland, G., and Elitzur, M. 1984, Ap. J., 285, L11.

Gondhalekar, P., O'Brian, P., and Wilson, R. 1986, M.N.R.A.S., 222, 71.

Peterson, B. M., et al. 1985, Ap. J., 292, 164.

Puetter, R. C. 1986, B.A.A.S, 18, 926

Puetter, R. C. 1988, preprint.

Voit, G.M., and Shull, J. M. 1988 preprint.

Zheng, W. et al. 1987, preprint. 